\title{
Matrixes of unconventional micro-optical components molded with etched silicon
}

Jorge Albero

jorge.albero@femto-st.fr

Christophe Gorecki

Lukasz Nieradko

Birgit Päivänranta

Virginia Gomez

Hugo Thienpont

Nicolas Passilly
Département MN2S, Institut FEMTO-ST, CNRS UMR 6174, Université de Franche-Comté, 25044 Besançon, France

Département MN2S, Institut FEMTO-ST, CNRS UMR 6174, Université de Franche-Comté, 25044 Besançon, France

Département MN2S, Institut FEMTO-ST, CNRS UMR 6174, Université de Franche-Comté, 25044 Besançon, France

Department of Physics and Mathematics, University of Joensuu, P.O. Box 111, FI-80101 Joensuu, Finland

Department of Applied Physics and Photonics, Vrije Universiteit Brussel, Pleinlaan 2, 1050 Brussels, Belgium

Department of Applied Physics and Photonics, Vrije Universiteit Brussel, Pleinlaan 2, 1050 Brussels, Belgium

Département MN2S, Institut FEMTO-ST, CNRS UMR 6174, Université de Franche-Comté, 25044 Besançon, France

This paper reports on a process to create microlenses characterized by unconventional footprints, spherical profiles and a wide range of sizes. Fabricated shapes such as squares, rectangles, ellipses, triangles and hexagons are tested alone as well as in matrix with high fulfill factors. The technique is based on molds from which microlenses are fabricated by UV-molding replication. The molds are produced by silicon wet isotropic etching in an acid solution. The process is mainly steered by temperature and etching concentration. The use of the proposed technology opens a wide range of geometries allowing the fabrication of microlenses matrices with high fulfill factors as well as microlenses for beam-shaping. [DOI: 10.2971/jeos.2010.10001]

Keywords: micro-optics, microlenses, microlens array

\section{INTRODUCTION}

The conventionality or non-conventionality of a component is mainly a subjective issue. Over the years, things that were called at first unconventional have become conventional or just obsolete. The term unconventional in optics is often used for qualifying unconventional combinations of conventional optical devices and components [1], to refer to the use of materials which are not usually intended to be used in the fabrication of optics [2], or even to talk about unconventional imaging and optical processing [3,4]. In this paper, the word unconventional refers to the unusual shape of the optical elements themselves, as it has been employed for instance in laser technologies mainly for beam shaping based on refractive or diffractive optics $[5,6]$. Refractive micro-optics having different footprint shapes than circular can also be found in the literature $[7,8,9,10,11,12]$. However, none of them are based on replication of molds etched on silicon, which is particularly efficient. It can be noted that etched silicon used as a mold is up to now a technique mostly dedicated to regular (i.e. with circular footprint) refractive optics $[13,14]$ except when it has been employed to fabricate the components themselves, for instance as mirrors with non conventional shapes [15]. Unconventional refractive microlenses are mostly interesting when they are fabricated as a matrix. Indeed, it allows increas- ing the fulfill factor compared to circular shaped lenses, understanding the fulfill factor as the ratio between the surface covered by the microlenses (i.e. efficient surface) and the total surface. High fulfill factors cover most of the surface to benefit from as much incident light as possible, in order to improve light efficiencies. Arrays of microlenses with minimal gaps are interesting for applications as light collection for CCD arrays, by focusing light that would otherwise have fallen onto the non-sensitive areas or as enhancement of the optical intensity output in laptop displays [16]. In particular, triangular microlenses arrays are of interest for LCD applications where they might be more efficient than the conventional diffuser films and brightness enhancement films [8].

In this study, we consequently propose the fabrication of unconventional refractive microlenses based on a recently developed technique for circular microlenses [14]. This technique is based on isotropic wet etching of silicon to produce molds from which optical elements are replicated by well known techniques of micromolding. The replication processes can vary depending on the material chosen for fabrication, whereas the common key step of the technology is the isotropic wet etching of silicon in acid solution used to pro- 
duce the wafer-scale molds. This technique allows the production of high quality surface molds at rather low-cost. In addition, high fulfill factors, difficult to obtain by other techniques are achievable [17]. Production of unconventional footprints is reached thanks to specific mask design which, combined with time of etching, concentration of etchant and temperature, determine the final shape. The wet isotropic etching of silicon is theoretically characterized by an etch ratio independent of the orientation of silicon crystallographic planes [18, 19, 20]. This feature allows fabricating molds that keep spherical profiles while the shape of their footprints depends on the initial shape and size of the mask and on etching solution parameters (temperature and concentration). Once the molds are ready, the next step consists in their replication. The advantage of this process is that a single etched silicon wafer containing the molds can be used to produce a large number of optical components. Replication leads to the final microlenses and is the step that demonstrates the ability of the technique to generate micro-optical elements. Various techniques can be used for the replication with similar results, such as hotembossing or micro-injection molding [21, 22, 23], however we chose in this study the solvent assisted UV-molding process [24], since it is more convenient for prototyping. Indeed, mold casting can be done directly without the need for intermediate tougher shims [25]. Then, this paper shows how this technique is able to produce various types of unconventional refractive microlenses in matrix or not, at a rather low-cost. For instance, it can lead to elliptical lenses, useful in order to shape edge emitting diode beams which are usually astigmatic [10]. Cylindrical microlenses, which can be used in 3D screens development and beam shaping for laser printers and line scanners [26], are also presented.

The paper is then organized as follows. Section 2 details the fabrication and characterization of the molds. In particular, we discuss the procedure of protecting the substrate with a hard mask, the development of the initial footprints of microlenses through the mask and the wet etching procedure to obtain the final molds. After that, the molds are characterized separately according to the shapes. The replication step is then addressed in Section 3 where fabrication and characterization of unconventional polymer microlenses are presented. Finally, conclusions and perspectives are given in Section 4.

\section{MOLDS FOR UNCONVENTIONAL MICROLENSES}

\subsection{Molds fabrication}

The first step of mold fabrication is the design of the mask. This design has been drawn with commercially available CAD software. At this point, the considerations for footprint determination concern the etch ratios which depend on concentration and temperature of the chosen etching solution. These data are necessary to calculate the mask features in order to obtain the desired size and shape of the microlenses [27, 28]. In our tests, the different footprints set in the mask are circles, squares, rectangles, hexagons, triangles, ellipses and circular crowns.

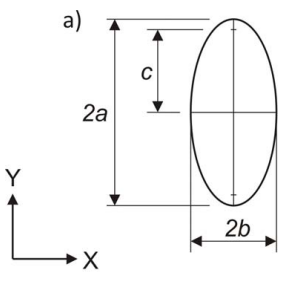

d)

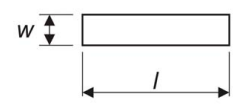

b)

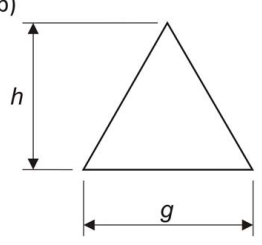

e)

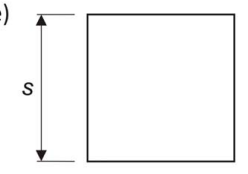

c)

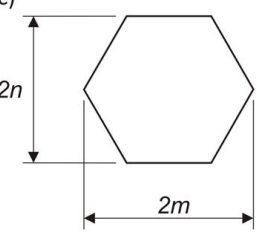

f)

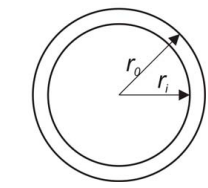

FIG. 1 Nomenclature used for the geometric characteristics of the designed footprints for the molds and axis directions used to characterize etch rates.

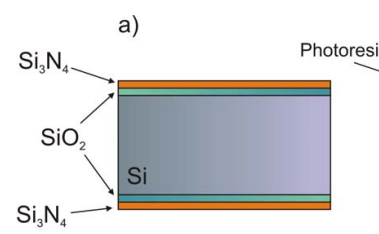

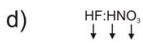

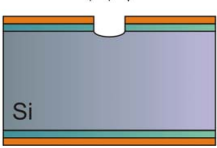

b) uv Mask

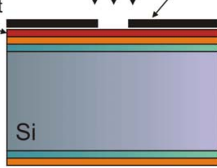

e)

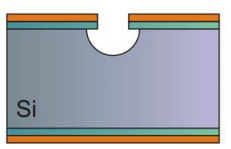

c)
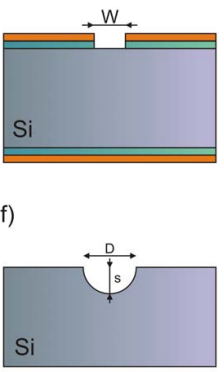

FIG. 2 Silicon etching process used for the fabrication of the molds by isotropic etching

The nomenclature used to designate their parameters is shown in Figure 1. Concerning the matrixes, sizes of elements and pitches between them are chosen depending on the etch rates resulting from the processes described in [14], the aim being to adapt the design in order to achieve high fulfill factors. Such shapes are chosen for different reasons: while circles lead to conventional spherical microlenses, ellipses (Figure 1(a)), providing ellipsoidal microlenses, can be used for instance for astigmatism correction. Triangles (Figure 1(b)) and hexagons (Figure 1(c)) are more suitable to achieve high fulfill factors when arranged in a matrix. Then, rectangles (Figure 1(d)) are tested in order to obtain cylindrical microlenses. Squares (Figure 1(e)) are geometrically suitable to attain high fulfill factors and the pitch between them will be a central point of interest of the study. Without any concrete application, circular crowns (Figure 1(f)) were chosen to demonstrate that most kinds of shapes able to be designed on the mask can be transferred to the mold (and then replicated), with the limitation of perfectly square corners inherent in the nature of the isotropic effect.

Once the mask is designed, the mold fabrication follows the process shown in Figure 2. A $\langle 111\rangle$ oriented silicon wafer is protected on both sides with a hard mask. The latter is made of $0.5 \mu \mathrm{m}$ of $\mathrm{SiO}_{2}$, grown by thermal oxidation, on which $100 \mathrm{~nm}$ of super-low stress $\mathrm{Si}_{3} \mathrm{~N}_{4}$, is deposited by low pressure chemical vapor deposition (LPCVD) (Figure 2(a)). Super-low stress $\mathrm{Si}_{3} \mathrm{~N}_{4}$ is mandatory in order to reduce the risk of broken parts in the zones of underetching. The hard mask is meant to protect the surface of the silicon wafer which should not be attacked while etching through the openings. To create these openings, the $\mathrm{Si}_{3} \mathrm{~N}_{4}$ layer was first spin coated with positive photoresist and the mask pattern was then transferred onto 
the surface by standard photolithography (Figure 2(b)). Afterwards, both $\mathrm{Si}_{3} \mathrm{~N}_{4}$ and $\mathrm{SiO}_{2}$ on the uncovered surfaces were removed by reactive ion etching (RIE) in order to create the apertures (Figure 2(c)).

The silicon wafer is consequently ready to be etched. The chosen etching solution to create the molds is a mixture of $\mathrm{HF} / \mathrm{HNO}_{3}$ in proportion 1:9, which acts as an isotropic etchant of silicon (Figures 2(d) and 2(e)). Wet isotropic etching of silicon is based on the theoretically constant etching speed irrespective of the crystallographic planes orientation of silicon $[18,19,20]$, unless practical effects modify this behavior $[29,30]$. The mechanism of $\mathrm{HF} / \mathrm{HNO}_{3}$ etching is the combination of two main effects. First, $\mathrm{HNO}_{3}$ causes the oxidation of the silicon surface, and secondly HF removes the generated oxide [31]. To produce the mold, the substrate is immersed in a Teflon container containing the solution at room temperature $\left(22^{\circ} \mathrm{C}\right)$ for 30 minutes and straight afterwards is rinsed in deionized water and heated up to $120^{\circ} \mathrm{C}$ in order to dry the surface. Then, the hard mask is removed with a solution of HF at $49 \%$ (Figure 2(f)). Differences in etching speeds have been observed experimentally between the bottom of the mold and the region under the mask. This was attributed to several effects such as masking from reaction residues (in the bottom) or presence of gases acting as catalyst (under the mask) resulting in a lower etch ratio at the central zone. This problem was solved by applying hand mixing of the solution while etching. It avoids flattened and convex surfaces at the bottom of the molds and ensures sphericity of the mold. For this type of silicon etching, the microlens maximum size is a limiting parameter in order to obtain spherical profiles. Another important aspect is the quality of the mold regarding the fidelity of the transferred shapes. It is actually significantly influenced by the orientation of the silicon substrate i.e. $\langle 111\rangle$ orientation avoids anisotropy related issues such as flattened-like sides of the footprint when elements with rounded zones are created [29].

\subsection{Molds characterization and evaluation}

The size characterization of the silicon molds was performed with both a non-contact profilometer (Wyko NT2000) and a SEM microscope, whereas the surface roughness was measured with a non-contact optical profilometer from Fogale. The latter measurements gave values spread between $4 \mathrm{~nm}$ and $6 \mathrm{~nm}$, demonstrating the good quality of the molds for optical components production. These results are better than those generally obtained from other fabrication techniques [9]. In the following characterization of the sizes, each shape is considered separately. It is important to underline that, when dealing with elliptical, triangular and hexagonal footprints, the shapes of the molds tend to become circular along with time, as if the original footprint were a circle. In order to quantify that effect, let us have a look at how the shape evolves in each case.

Concerning elliptical shapes (Figure 3(a)), the elliptical relation defined as $2 a / 2 b$ (Figure 1(a)) gives us an idea a priori of the evolution of the shapes resulting from etching. This relation is plotted in Figure 4(a) as a function of the major axis $2 a$ on the mask and for all different tested values of the minor

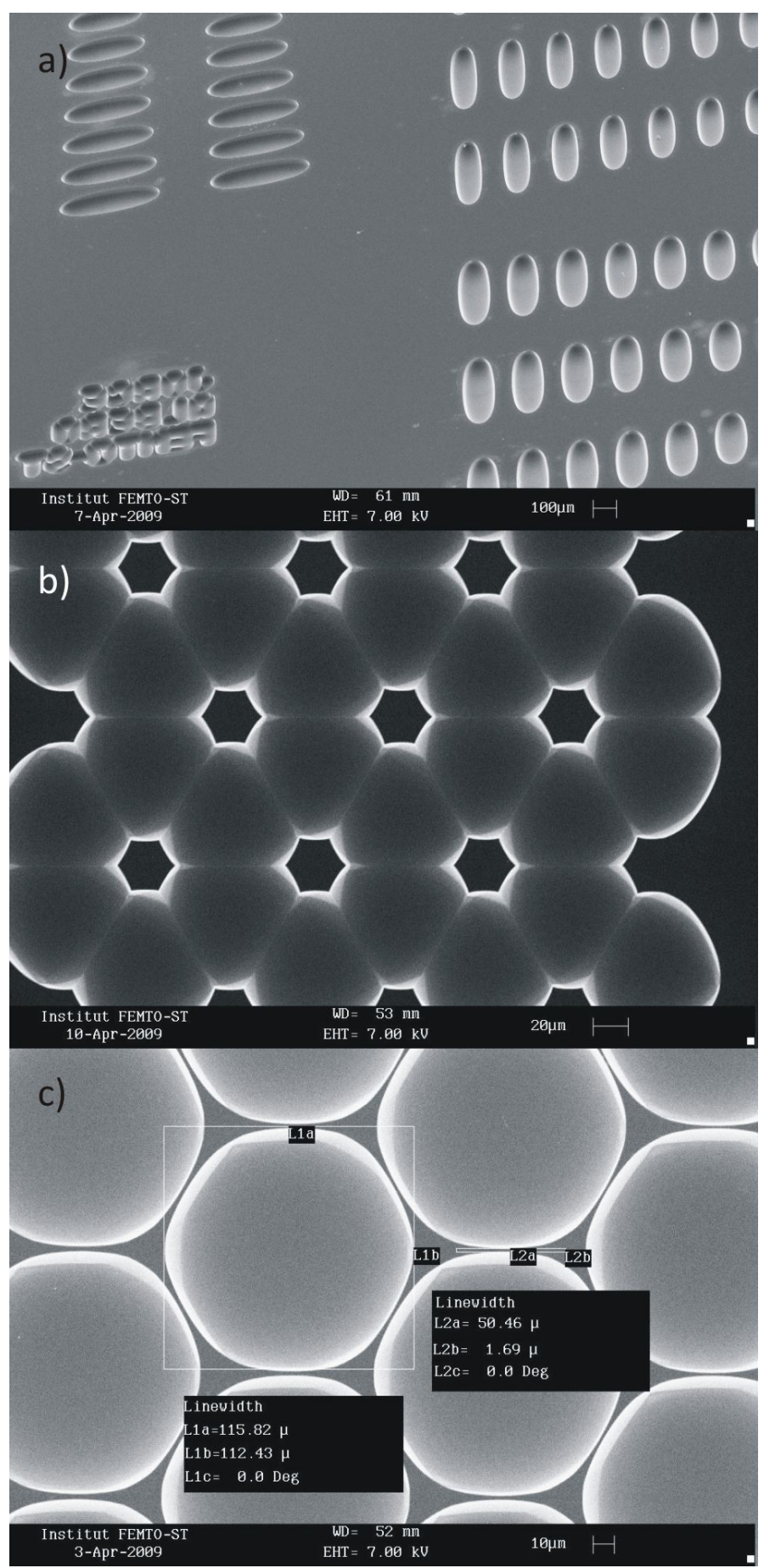

FIG. 3 SEM images of the different molds, all with spherical profile but different footprints, (a) elliptical shapes with different major and minor axis length, (b) matrix of triangular-like shapes of $70.3 \mu \mathrm{m}$ height and $78.8 \mu \mathrm{m}$ base, (c) matrix of hexagonal-like shapes with $2 m=115.82 \mu \mathrm{m}$ and $2 n=112.43 \mu \mathrm{m}$.

axis $2 b(30,50$ and $70 \mu \mathrm{m})$. The plotted values, corresponding to patterns before and after etching, are in all cases higher on the mask than on the mold, i.e., the ellipticity is always reduced when the mold is created. In addition, this reduction is accentuated for shorter minor axis $2 b$. Thus, as the values of the minor axis $2 b$ increase, the etching speeds on each axis direction become more and more similar, leading to a shape in better agreement with the original design of the footprint.

The etching speed actually depends on the width of the opening. For the shortest minor axis, i.e., when the space between 

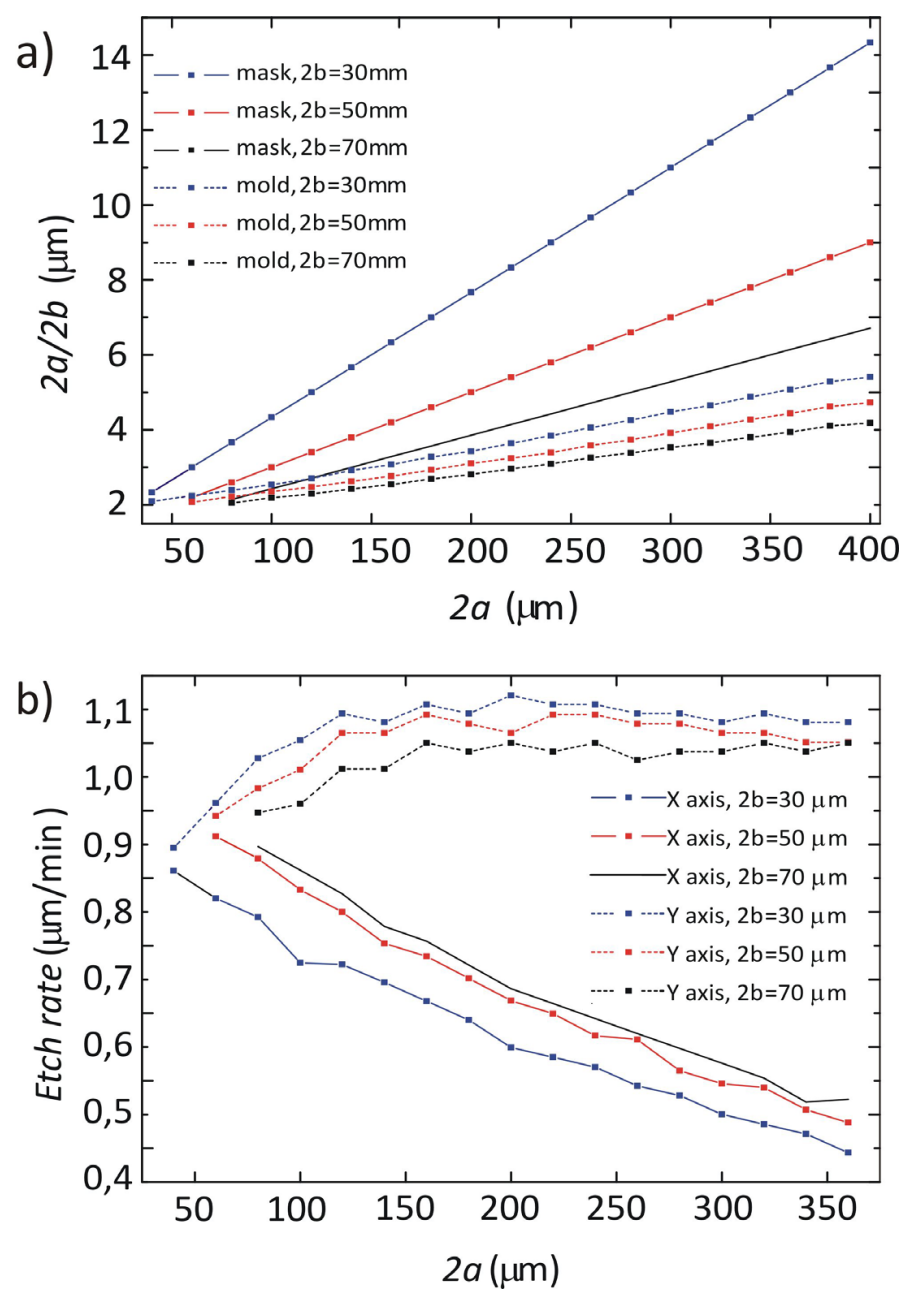

FIG. 4 (a) Elliptical relation $2 a / 2 b$ vs. major axis size, (b) etch rates of the ellipsoidal molds depending on the axis orientation vs. the major axis size.

sidewalls is narrower, the etching speed along the $Y$ axis (defined in Figure 1) is reduced significantly compared to the speed along the $X$ axis (Figure $4(\mathrm{~b})$ ), and the difference increases as the major axis gets longer. Eventually, the footprint tends to become a circle after etching. This is understandable since the mass transfer is enhanced when the circulation of reactants into the mold gets easier as it does when reaching the edges of the major axis. This effect is clear also if we look along the $Y$ direction for a given value of the major axis $2 a$, the etch rate increases along with increases in the minor axis. However, it seems that the etching speed along $X$ is not influenced by increasing of the major axis length $2 a$, since it remains nearly constant (Figure 4(b)). It can then be stated that the etch rate is more sensitive to small distances between sidewalls.

To study the equilateral triangles (Figure 3(b)), the sizes of base and height of the footprints as a function of the mask base size are plotted in Figure 5(a). The base is measured between one apex and the adjacent one, whereas the height is taken from the third and remaining apex to the center of the opposite edge. The values are represented before (mask) and after etching (mold) for the matrixes having a pitch between items of $p=60 \mu \mathrm{m}$. By definition, height of equilateral triangles is always $15.5 \%$ longer than their base. This feature can be seen on the plot of height and base size of the mask pat-
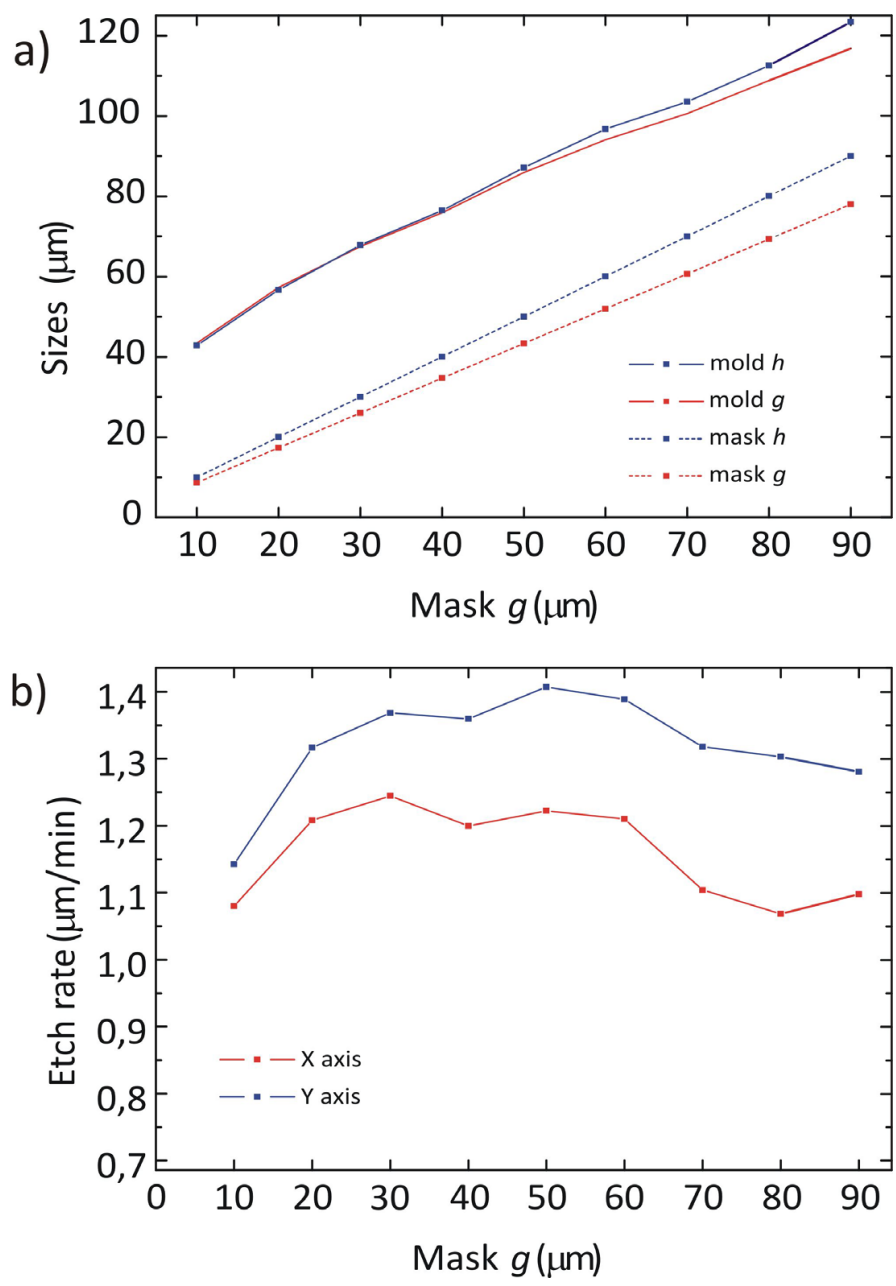

FIC. 5 (a) on the triangular footprint matrix with pitch $p=60 \mu \mathrm{m}$, comparison of the sizes of base $g$ and height $h$ before and after etching vs. the mask base size, (b) etch rates of the triangular molds depending on the axis orientation vs. the mask base size.

tern on the Figure 5(a). However, after etching, this proportion changes and the height is almost equal to the base in most cases. This effect is due to a circularization of the footprint, as shown in Figure 6(a). Indeed, if we look at the etch rates along $X$ and $Y$ directions (as specified on Figure 1), plotted on Figure 5(b), the etch rate along $X$ direction is slower than along $Y$ direction, independent of the base size. This is due to the action of the etchant (mass transfer) which gets easier against a straight edge than enclosed in a vertex. Thus, due to consecutive differences of the etch rate, the straight edge tends to bend while the apex tends to circularize leading to a deterioration of the triangular shape. It can be noted that similar behavior was observed for matrixes having different pitches between items ( $p=60$ and $80 \mu \mathrm{m})$.

Concerning the hexagonal footprints, their investigation is done through the study of the ratio of the circumscribed diameter $2 m$ over the inscribed diameter $2 n$ (Figure $7(\mathrm{a})$ ). The ratio $2 m / 2 n$ for a regular hexagon as designed on the mask is 0.866 . On the Figure 7(a), ratios measured on single hexagonal molds or disposed in matrix are in all cases higher after etching, especially when the size of the hexagon decreases. In the latter case, the value of $2 m / 2 n$ is near to 1 , as for a circularlike shape (Figure 6(b)). From Figure 7(b), it can be deduced that the same behavior exists as it does for triangles: the in- 


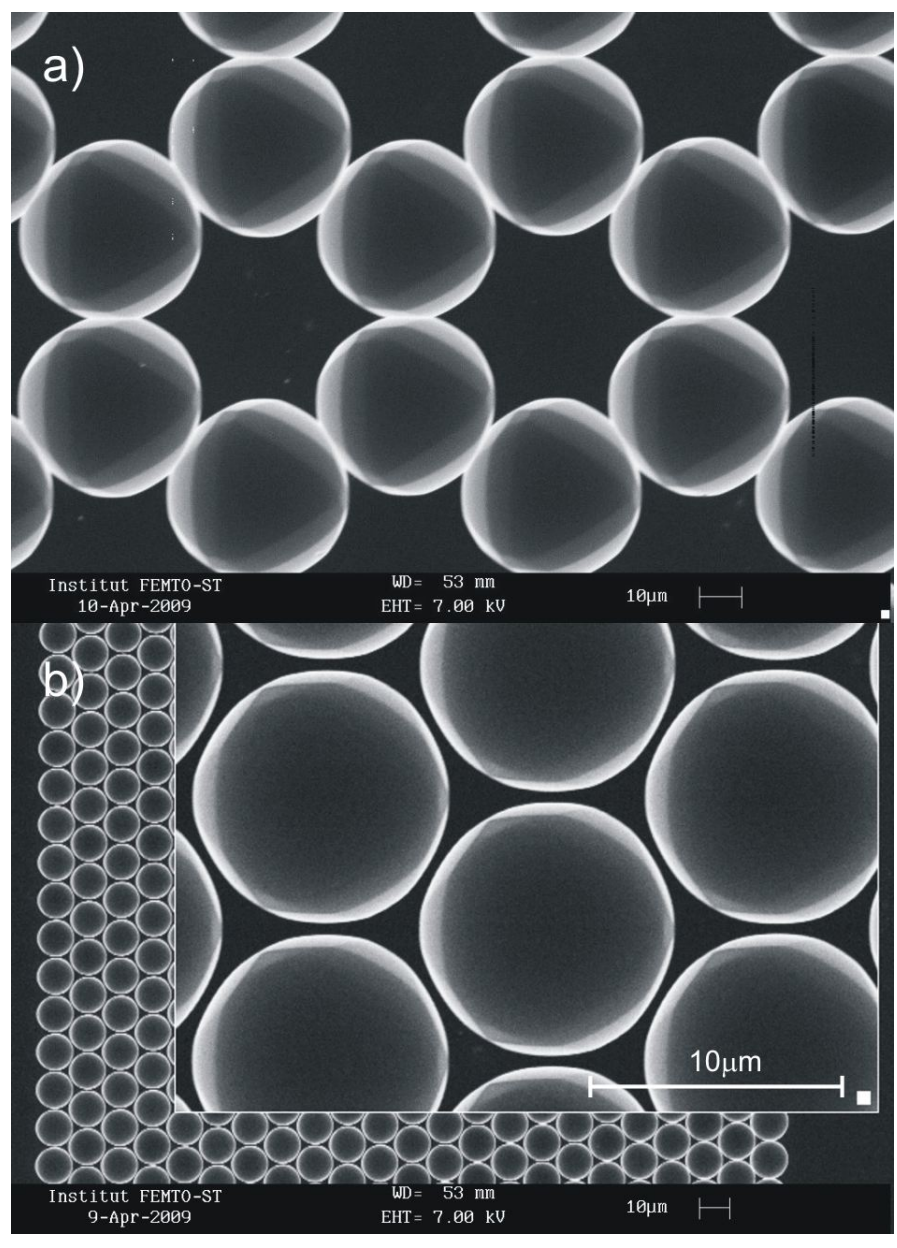

FIG. 6 Over-etching of the designed shapes, (a) triangles coming up to circles and (b) hexagons coming up to a circle.

scribed diameter, measured between two opposite edges, increases quicker than the circumscribed diameter, measured between two opposite vertices. Then, the etch rate in $Y$ direction is higher than the etch rate in $X$ direction.

It is important to underline that the etch rate is significantly dependant on the molds disposition. Indeed, as it can be seen on the Figures 7(a) and 7(b), the etch rates of the single hexagons are usually higher than those corresponding to matrixes of hexagons. Small pitch seems to reduce even more the etch rate. This is expected to be due to the so-called loading effect studied by Kuiken et al. [32]. Thus, the vicinity effect is relevant to the etching speed and needs to be taken into account.

Another tested geometry is the squared one. Again, a similar effect has been observed, i.e., bending of edges surrounded by corners. It has to be mentioned that the latter effect can limit the achievement of very high fulfill factor matrixes. In this work, the square shapes were designed in order to maximize the fulfill factor for a given time of etching (30 minutes). The obtained matrix shown in Figure 8(a) has a fulfill factor in the order of $85 \%$ (ratio of surfaces) that is expected to be improvable by fine optimization. Indeed, if we try to extrapolate what would be the maximum fulfill factor when edges of the squared or hexagonal shapes are in contact (i.e., the straight edges since they are the ones bending), we can estimate the maximum fulfill factor to be around $92 \%$, whereas $90 \%$ seems

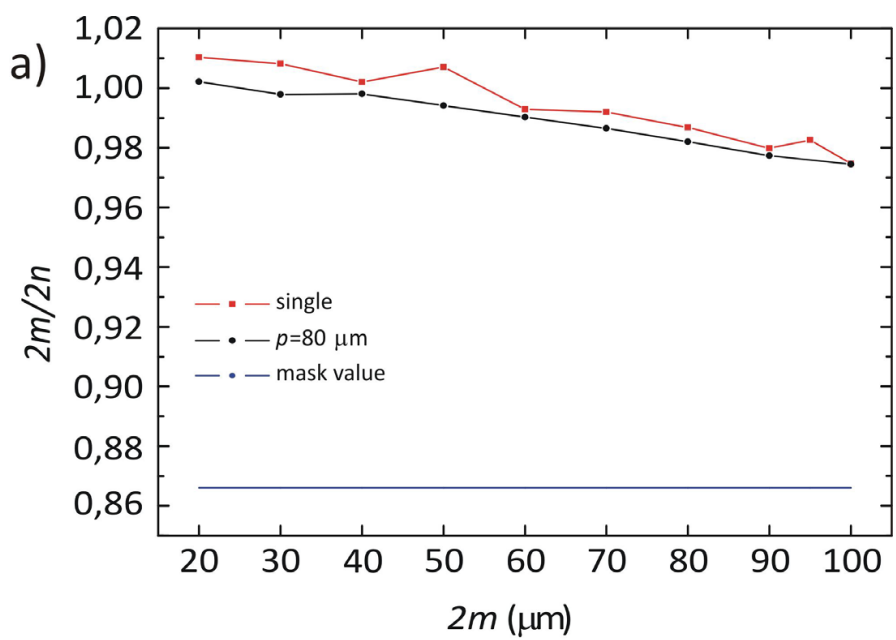

b)

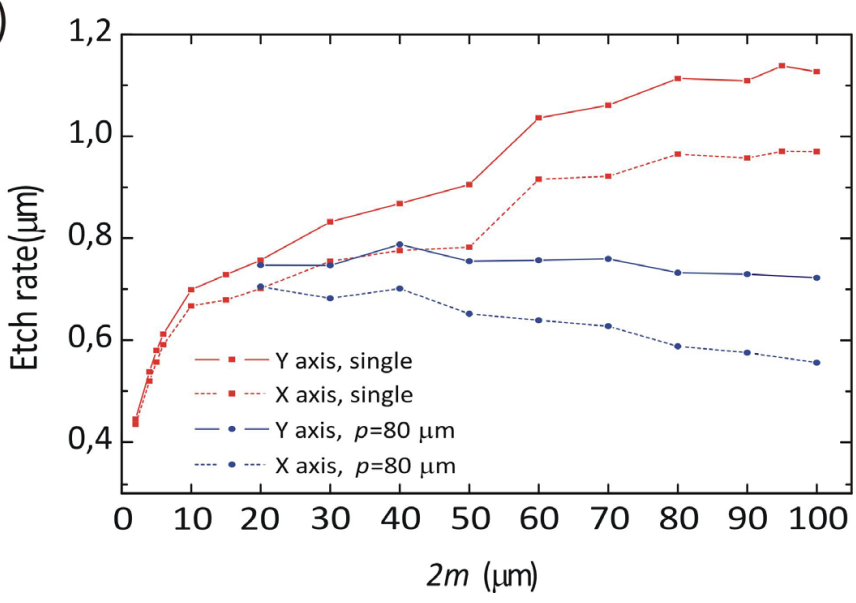

FIC. 7 (a) ratio $2 m / 2 n$ of the hexagonal footprints on the mask, after etching the single molds and in matrix with pitch $p=80 \mu \mathrm{m}$ vs. the value of $2 m$ on the mask and (b) etch rates of the single hexagonal footprint molds and in a matrix with pitch $p=80 \mu \mathrm{m}$ depending on axis orientation vs. the value of $2 m$ on the mask.

to be the maximum value for triangular shapes. Consequently, the shapes onto the mask should slightly differ from pure triangles, squares and hexagons in order to compensate the circularization and then achieve nearly $100 \%$ fulfill factor.

In the case of cylindrical microlenses, different lengths have been tested. Deviations from the initial rectangular shape are noticed at the extremities which become rounded (for the same reasons as previously) (Figure 8(b)). However, when the length $l$ is long enough, the silicon is attacked homogeneously by the etchant solution and measured deviations of width $w$ are in all cases under $1 \mu \mathrm{m}$. The cylindrical shape is maintained with constant depth and curvature all along the microlens without length limitation (lengths up to $13 \mathrm{~mm}$ have been realized). In matrix configuration, Figure 9 shows that a $100 \%$ fulfill factor can be achieved. Finally, Figure 8(c) shows how circular crowns can be arranged when dealing with diameters and positions.

From these different characterizations, we can conclude that whatever the footprints on the mask are, the etching has to be limited in time to maintain the general shape of the design (elliptical, triangular, etc.) and to avoid strong circularization. Especially for the smallest footprints, the mold can quickly circularize and end up as a standard spherical microlens. In 

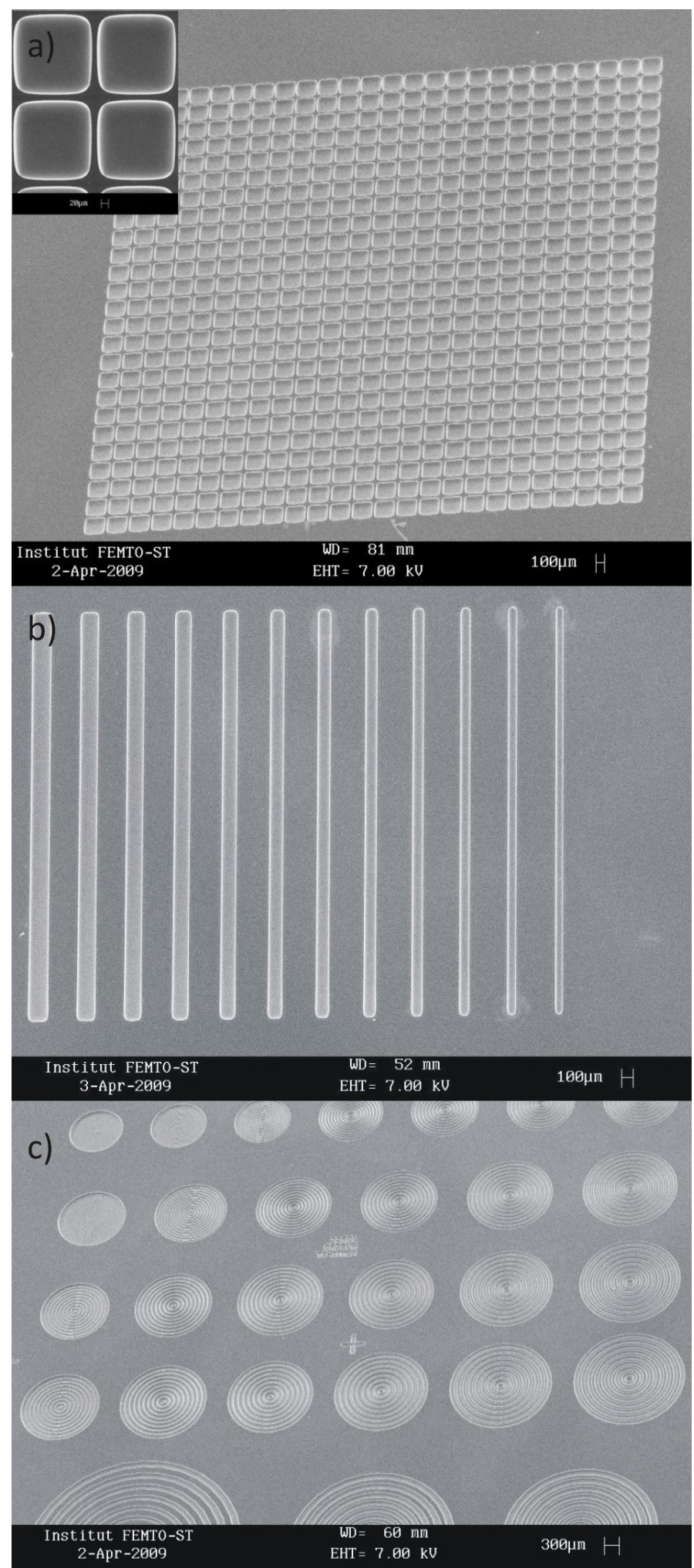

FIG. 8 SEM images of the different molds, all with spherical profile but different footprints, (a) matrix of square-like shapes of $220 \mu \mathrm{m} \times 220 \mu \mathrm{m}$ measured at the most distant points of the edges, (b) rectangular-like shapes to form cylindrical microlenses of $996.18 \mu \mathrm{m}$ long and different widths, and (c) circular crowns of different sizes.

addition, it has to be taken into account that etch rates depend on the pattern when the components are disposed in matrix. Thus, bending of straight edges between corners and apex needs to be anticipated in order to achieve high fulfill factors.

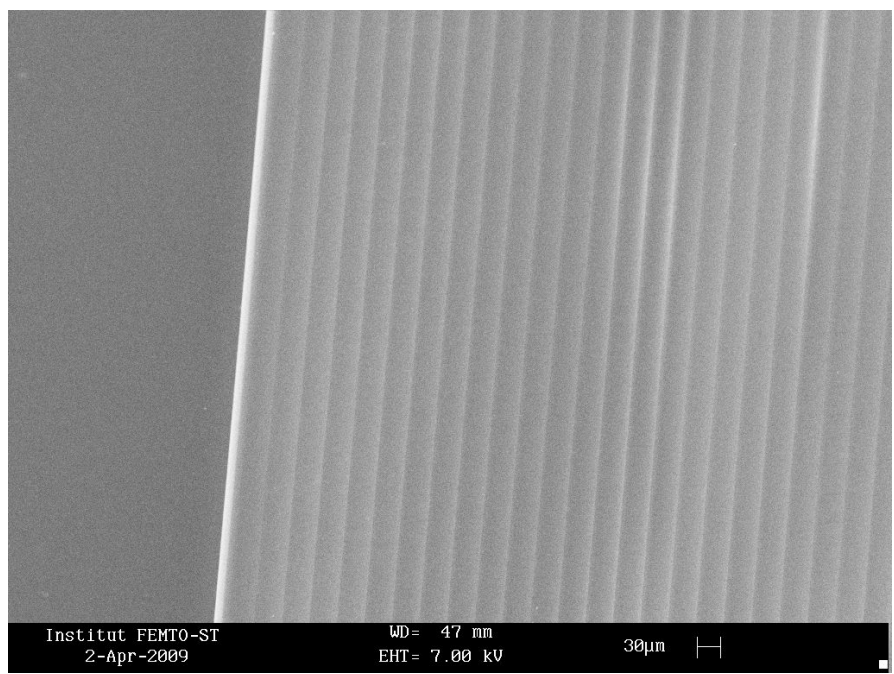

FIG. 9 Rectangles etched enough long to be a $100 \%$ fulfill factor matrix

\section{UNCONVENTIONAL MICROLENSES FABRICATION}

\subsection{Molds replication: microlenses fabrication}

The replication of the polymer microlenses from the silicon molds was performed by heat and solvent assisted UVmolding, method introduced by Pietarinen et al. [24] to avoid air trapping and promote high fidelity of shapes. Other commonly used methods such as micro-injection molding or hotembossing are also expected to be suitable regarding results from [14], but were not considered in this study since they are not so practical for prototyping (due to the necessity of an intermediate Nickel shim in order to avoid applying high forces directly to the silicon molds). The reasonable simplicity of the method, the fidelity of the replicas with respect to the molds and the repeatability of the replication process from the same mold are the key points that make UV-molding a reliable choice to fabricate the microlenses.

The material used to replicate the molds was SK9 from Summers optics, a UV-curable optical cement with high transparency in the visible wavelengths. Prior to replication, antiadhesive treatment is applied to the mold. For this purpose, the mold is coated with a $5-10 \mathrm{~nm}$ layer of $\mathrm{SiO}_{2}$ and treated with a liquid-phase deposition of alkyltrichlorosilane to modify the surface energy, i.e., promote the separation of the molded part from the master. Then, the mold is filled with an easily evaporable solvent and the liquid SK9 (pre-polymer) is immersed through the solvent film on the master structure. By heating the whole set up to $80^{\circ} \mathrm{C}$, the solvent dissolves the pre-polymer to fill the entire master and, at the same time, the solvent evaporates away from the top of the pre-polymer surface. Finally, the steps of UV-curing and de-molding are performed as in a basic UV-molding process.

\subsection{Optical characterization}

We measured the micro-lenses focal length, considering it as the plane where the energy concentrates in the smallest possible area across the optical axis. The setup used for the 

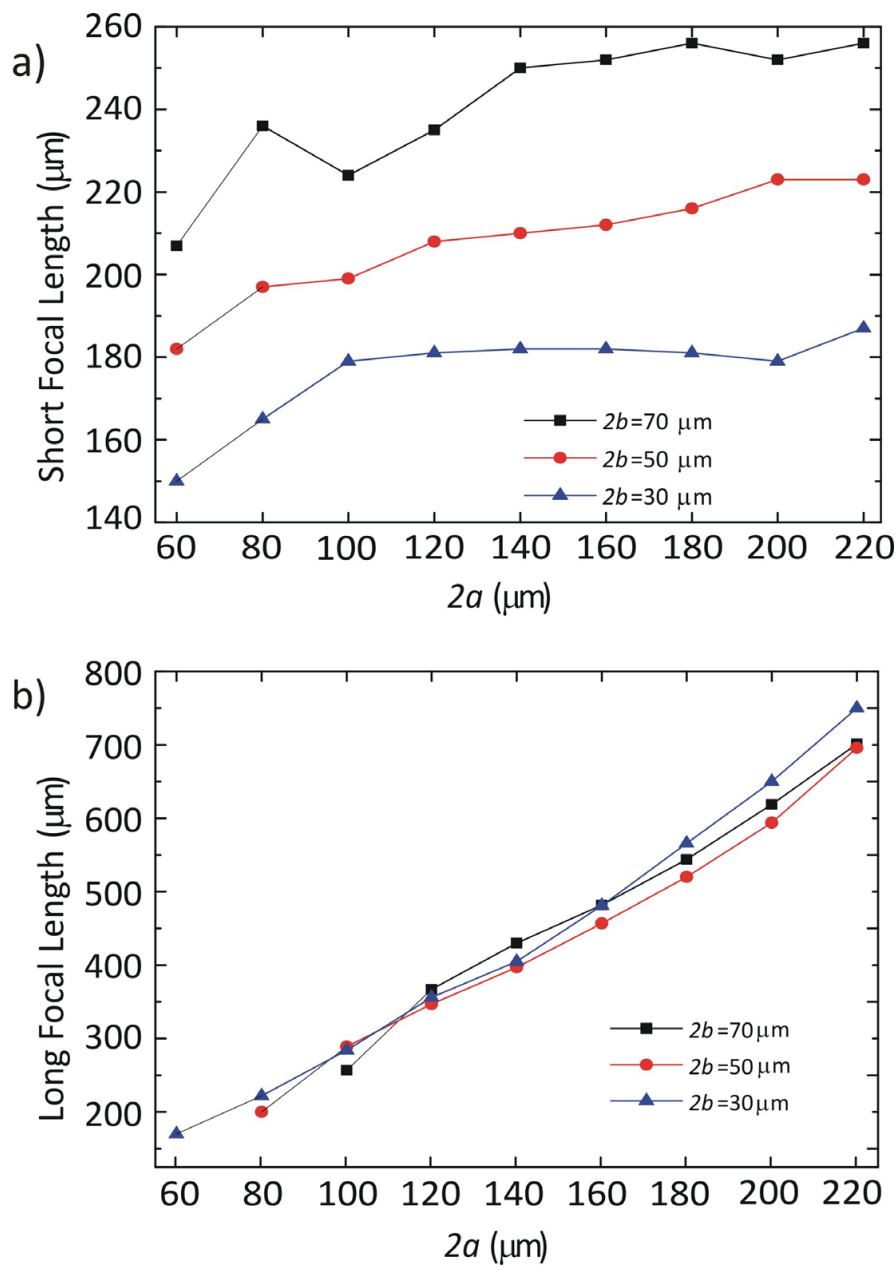

FIG. 10 (a) Measured "short" focal length of the ellipsoidal microlenses for the three different values of the minor axis $2 b(30,50$ and $70 \mu \mathrm{m})$ vs. major axis $2 a$ on the mask, (b) measured "Iong" focal length of the ellipsoidal microlenses for the same values of $2 b$ as in (a) vs. major axis $2 a$ on the mask.

measurements consisted in a collimated laser beam $(\lambda=$ $632.8 \mathrm{~nm}$ ) passing through the microlenses before being imaged by a microscope objective in a CMOS camera. The incident beam is collimated so that the focal length refers to the position of the lens focal plane.

Regarding the ellipsoidal microlenses, we measured the socalled "short" focal length (SFL) and "long" focal length (LFL) [10] as a function of the major axis (Figure 10(a) and 10(b), respectively). Looking at Figure 10(a), the SFL is mainly constant for a given value of the minor axis $2 b$ independent of the size of the major axis $2 a$. Figure 10(b) shows the complementary effect for LFLs, where they increase along with $2 a$ but keep constant with $2 b$. Then, the SFL and LFL are only dependent on respectively $2 b$ and $2 a$. We should be consequently able to fabricate a dedicated ellipsoidal microlens to collimate astigmatic beams whatever their relation is. An image taken at the SFL can be seen on Figure 11(a). An example of light line produced by one of the cylindrical microlenses is shown in Figure 11(b). This kind of beam shaping can be useful for sensor applications. Regarding the other microlens shapes, the focal distances of cylindrical microlenses and hexagonal footprint microlenses behave linearly with the width $w$ and the circumscribed diameter $2 m$, respectively (Figures 12(a) and 12(b)). The obtained linear behavior is con-
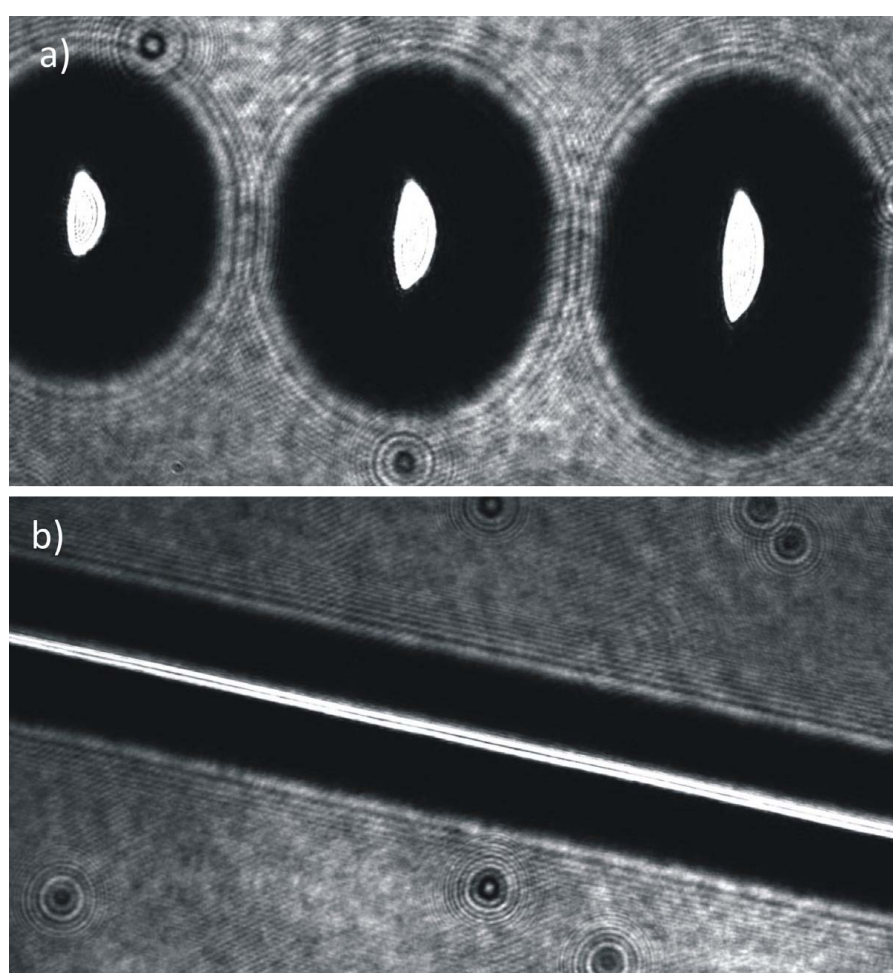

FIG. 11 (a) Light intensity through ellipsoidal microlenses having different ellipticities (left to right, 1:2.2, 1:2.5, 1:3.1) and (b) light line produced by a cylindrical microlens.

venient for design considerations. In addition, the numerical aperture of hexagonal microlenses can be estimated from experimentally measured diameters and focal lengths using the following approximation:

$$
N A=n \sin \theta=\frac{d / 2}{\sqrt{f^{\prime 2}+\left(\frac{d}{2}\right)^{2}}} .
$$

where $n$ is the index of refraction of the medium in which the microlens is immersed, $\theta$ is the acceptation angle, $d$ is the diameter of the microlens and $f^{\prime}$ its focal length. When the inscribed diameters $2 n$ of the different hexagonal microlenses are taken into account, numerical apertures between 0.30 and 0.37 have been obtained.

Being one of the most widely used for high fulfill factor matrixes, the hexagonal footprint microlenses are chosen in here to study the optical resolution with a USAF 1951 target. Figure 13 shows the images of two hexagonal footprint microlenses imaging the smallest group of a $2^{\prime \prime} \times 2^{\prime \prime}$ USAF 1951 target for standard resolution. In both cases, the smallest pattern of the target, with 228 cycles per $\mathrm{mm}$, i.e., lines or spaces of $2.2 \mu \mathrm{m}$ wide are clearly imaged. The measured magnification of the microlens shown in Figure 13(a) is around 5.1×, whereas for the microlens in Figure 12(b) is around $1.8 \times$. It can be noted that the distortion is low in most of the microlenses area.

\section{CONCLUSION}

We present in this paper a technique based on isotropic wet etching dedicated to the fabrication of silicon-molds for microlenses production. We show that this technology can be 
a)

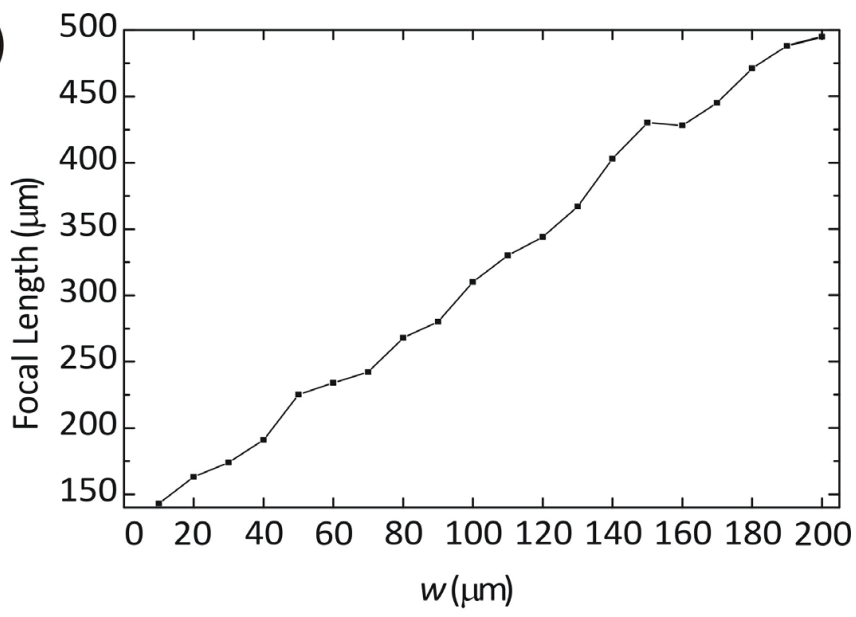

b)

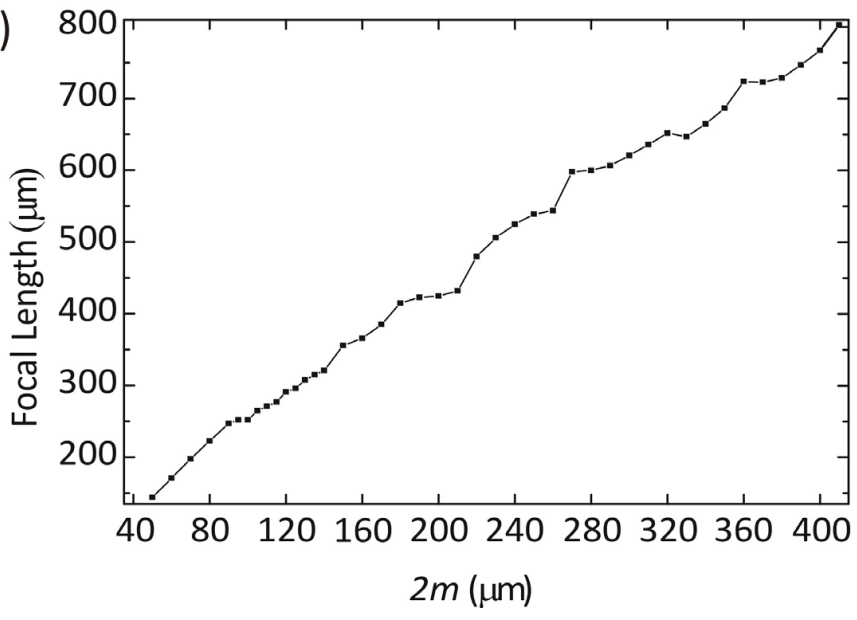

FIG. 12 (a) Measured focal distance of the cylindrical microlenses vs. the width of rect angles $w$ on the mask and (b) measured focal distance of the hexagonal microlenses as single items (not in matrix disposition) vs. the distance between opposite vertices $2 m$.
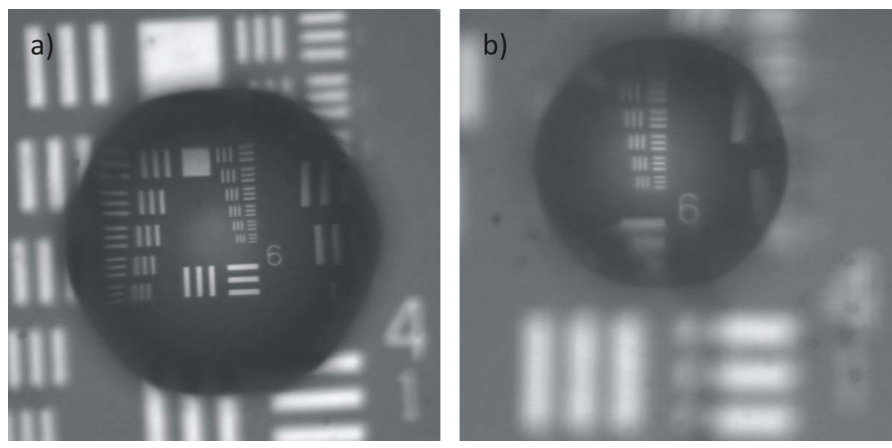

FIG. 13 Images of a USAF 1951 target through hexagonal footprint microlenses (single items) made by UV-molding of SK9 optical cement, a) diameter $2 n=464 \mu \mathrm{m}$, sag $=71 \mu \mathrm{m}$ and estimated $N A=0.30$, the image shows a good recognition of the smallest pattern, with 228 cycles per mm, b) diameter $2 n=310 \mu \mathrm{m}$, sag $=12.7 \mu \mathrm{m}$ and estimated $N A=0.33$, the same pattern as in a) can be also recognized. The areas on the target that can be imaged without distortion are respectively $250 \mu \mathrm{m}$ and $120 \mu \mathrm{m}$ wide.

applied to fabricate molds for microlenses of arbitrary footprints (triangular, hexagonal, cylindrical, etc.) and spherical profiles. The molds can be then replicated to fabricate arrays of lenses characterized by high fulfill factors by using conventional replication techniques such as UV-molding (tested in this paper), micro-injection molding, hot-embossing, glass re- flow, etc. From mold characterizations, it was observed that etching tends systematically to circularize the original shape of the footprints, due to bending of straight edges between corners which are moreover getting rounder. Thus, the etching time has to be reasonable and furthermore, in agreement with the disposition in the matrixes since it influences the etching rate. The measurements show resolutions better than $2.2 \mu \mathrm{m}$. Such technique is intended to be used in various optical applications. Amongst them: lab-on-chip detection for imaging small objects, applications where light collection efficiency is important (with the help of high fulfill factor matrixes) or laser astigmatism correction (in the case of elliptical microlenses). In all cases, the technique guarantees good reproducibility and compatibility with low cost production thanks to replication.

\section{Acknowledgements}

The authors want to acknowledge the support of the Network of Excellence on Micro-Optics (NEMO).

\section{References}

[1] W. Welford, and R. Winston, "Nonconventional optical systems and the brightness theorem" Appl. Opt. 21, 1531-1533 (1982).

[2] G. Petrovskii, "School of optical material science at the S. I. Vavilov State Optical Institute" J. Opt. Technol. 70, 855-856 (2003).

[3] K. lizuka, and M. Kawakita, "Unconventional Imaging" Opt. Photonics News 13, 61 (2002).

[4] E. Marom, N. A. Vainos, A. A. Friesem, and J. W Goodman, “Unconventional Optical Elements for Information Storage, Processing and Communications" (Proceedings of the NATO Advanced Research Workshop, NATO, Tel Aviv, 1999).

[5] S. Zhang, "A simple bi-convex refractive laser beam shaper" J. Opt. A - Pure Appl. Opt. 9, 945-950 (2007).

[6] N. Passilly, M. Fromager, L. Mechin, C. Gunther, S. Eimer, T. Mohammed-Brahim, and K. Aït-Ameur, "1-D Laser beam shaping using an adjustable binary diffractive optical element" opt. Commun. 241, 465-473 (2004).

[7] S. Hsiao, C. Lee, and W. Fang, "Novel concave-based micro optical components" (Proceedings of the IEEE 21st International Conference on Micro Electro Mechanical Systems, Institute of Electrical and Electronics Engineers, Tucson, pp. 124-127, 2008).

[8] C. T. Pan, and C. H. Su, "Fabrication of gapless triangular microlens array" Sensor. Actuat. A - Phys. 134, 631-640 (2007).

[9] M. -C. Chou, C. T. Pan, S. C. Shen, M. -F. Chen, K. L. Lin, and S. -T. Wu, "A novel method to fabricate gapless hexagonal micro-lens array" Sensor. Actuat. A - Phys. 118, 298-306 (2005).

[10] W. -Royall Cox, T. Cheng, and D. J. Hayes, “Micro-optics fabrication by ink-jet printing" Opt. Photonics News 12, 32-35 (2001).

[11] T. -H. Lin, H. Yang, C. -K. Chao, and S. -Y. Hung, “New high fill-factor triangular microlens array fabrication method using UV proximity printing" Microsyst. Technol. 12, 1255-1261 (2009).

[12] W. -H. Lee, and T. Özel, "Laser Micro-Machining of Spherical and Elliptical 3-D Objects using Hole Area Modulation Method" ICASE Working Paper 07-021 (2007). 
[13] K. P. Larsen, J. T. Ravnkilde, and 0. Hansen, "Investigations of the isotropic etch of an ICP source for silicon microlens mold fabrication" J. Micromech. Microeng. 15, 873-882 (2005).

[14] J. Albero, L. Nieradko, C. Gorecki, H. Ottevaere, V. Gomez, H. Thienpont, J. Pietarinen, B. Päivänranta, and N. Passilly, "Fabrication of spherical microlenses by a combination of isotropic wet etching of silicon and molding techniques" Opt. Express 17, 6283-6292 (2009).

[15] G. V. Vdovin, 0. Akhzar-Mehr, P. M. Sarro, D. W. De Lima Monteiro, and M. Y. Loktev, "Arrays of spherical micromirrors and molded microlenses fabricated with bulk Si micromachining" Proc. SPIE 4945, 107-111 (2003).

[16] B. Ezell, "Making microlens backlights grow up" Inform. Display. $17,42-45$ (2001).

[17] A. Tripathi, T. V. Chokshi, and N. Chronis, "A high numerical aperture, polymer-based, planar microlens array" Opt. Express 17, 19908-19918 (2009).

[18] H. Robbins, and B. Schwartz, "Chemical etching of Silicon I" J. Electrochem. Soc. 106, 505-508 (1959).

[19] H. Robbins, and B. Schwartz, "Chemical etching of Silicon II" J. Electrochem. Soc. 107, 108-111 (1960).

[20] B. Schwartz, and H. Robbins, "Chemical etching of Silicon III" J. Electrochem. Soc. 108, 365-372 (1961).

[21] X. J. Shen, L. Pan, and L. Lin, "Microplastic embossing process: experimental and theoretical characterizations" Sensor. Actuat. A - Phys. 97-98, 428-433 (2002).

[22] B. -K. Lee, D. S. Kim, and T. H. Kwom, "eplication of microlens arrays by injection molding" Microsyst. Technol. 10, 531-535 (2004).
[23] P. Huang, T. Huang, Y. Sun, and S. Yang, "Fabrication of large area resin microlens arrays using gas-assisted ultraviolet embossing" Opt. Express 16, 3041-3048 (2008).

[24] J. Pietarinen, V. Kalima, T. T. Pakkanen, and M. Kuittinen, “Improvement of UV-moulding accuracy by heat and solvent assisted process" Microelectron. Eng. 85, 263-270 (2008).

[25] J. Pietarinen, S. Siitonen, N. Tossavainen, J. Laukkanen, and M. Kuittinen, "Fabrication of Ni-shims using UV-moulding as an intermediate step" Microelectron. Eng. 83, 492-498 (2006).

[26] X. Zhang, Q. Tang, X. Yi, Z. Zhang, and X. Pei, “Cylindrical microlens array fabricated by argon ion-beam etching" Opt. Eng. 39, 3001 (2000).

[27] K. R. Williams, and R. S. Muller, "Etch rates for Micromachining Processing” J. Microelectromech. S. 4, 256-269 (1996).

[28] K. R. Williams, K. Gupta, and M. Wasilik, "Etch rates for Microma chining Processing - Part II" J. Microelectromech. S. 12, 761-778 (2003).

[29] V. B. Svetovoy, J. W. Berenschot, and M. C. Elwenspoek, "Experimental investigation of anisotropy in isotropic silicon etching" J. Micromech. Microeng. 17, 2344-2351 (2007).

[30] C. B. Shin, and D. J. Economou, "Forced and natural convection effects on the shape evolution of cavities during wet chemical etching" J. Electrochem. Soc. 138, 527-538 (1991).

[31] X. G. Zhang, Electrochemistry of silicon and its oxide, (Kluwer Academic/Plenum Publishers 2001).

[32] H. K. Kuiken, J. J. Kelly, and P. H. L. Notten, "Etching profiles at resist edges I. Mathematical models for Diffusion-Controlled cases" J. Electrochem. Soc. 133, 1217-1226 (1986). 